\title{
Technique
}

\section{Technical Strategies and Anatomic Considerations for an Extrapedicular Modified Inferior Endplate Access to Thoracic and Lumbar Vertebral Bodies}

From: University of Oklahoma College of Medicine, Oklahoma

City, OK

Address Correspondence: Scott Burner, BS University of Oklahoma College of Medicine 941 Stanton L. Young Blvd. BSEB Room 103 Oklahoma City, OK 73104 E-mail: scott-burner@ouhsc.edu

Disclaimer: There was no external funding in the preparation of this manuscript. Conflict of interest: Each author certifies that he or she, or a member of his or her immediate

family, has no commercial association (i.e., consultancies, stock ownership, equity interest, patent/licensing arrangements, etc.) that might pose a conflict of interest in connection with the submitted manuscript.

Manuscript received: 04-23-2015 Revised manuscript received: 05-06-2016

Accepted for publication: 05-16-2016

Free full manuscript: www.painphysicianjournal.com
Background: Percutaneous access to the vertebral bodies is commonly done via the transpedicular approach for both diagnoses and treatment of spinal pathology. While this approach is effective in most cases, it is difficult in certain situations such as a patient with obstructing hardware from prior surgery.

Objectives: To investigate and illustrate an alternative to the typical percutaneous access to the vertebral body via an extrapedicular approach and to determine the complications associated with this approach.

Study Design: Description of a novel percutaneous vertebral body access technique developed during cadaver dissection and a report of complication rates in cases that were performed using this technique.

Setting: Radiology department at a private institution.

Methods: An effective extrapedicular access technique that could safely and consistently allow the needle tip to be placed in the center of the vertebral body was developed from cadaver dissection observations for the purpose of clinical use. A total of 96 vertebral compression fractures from T5 to L5 were treated via the extrapedicular technique at our institution between July 2008 and August 2012. There were 72 patients between ages 27 and 98 (mean age 73.2 years) who underwent treatment.

Results: Cadaver dissection revealed a relatively avascular and aneural portion of the inferior vertebral body just anterior to the pedicle. A total of 96 vertebral fractures were treated using the extrapedicular technique without any recognized clinical complications from the needle access or the instrumentation.

Limitations: The trial included a relatively small sample size, representing 7.4 percent of total patients treated at our institution. This was likely the result of a smaller patient population with contraindications to typical transpedicular access.

Conclusions: The thoracic and lumbar vertebral bodies may be accessed using a percutaneous extrapedicular access technique which represents a relatively avascular and aneural approach to the vertebral body. The technique presented allows access to the vertebral body around existing hardware and can accommodate the placement of large instruments. This technique was not associated with any known complications in our series of patients.

Key words: Vertebral body, spine, extrapedicular, kyphoplasty, vertebroplasty, paraspinal, thoracic, lumbar

Pain Physician 2016; 19:593-601 
P ercutaneous access to the vertebral bodies has proven to be a vital technique for a multitude of procedures ranging from biopsies to treatment of hemangiomas and vertebral compression fractures (1-4). Transpedicular percutaneous biopsies were performed prior to the first vertebroplasty for the purpose of histologic analysis of the vertebral body and have been used consistently for vertebral augmentation since the late 1980s (5-7). The primary purpose of percutaneous access to the vertebral bodies today is for the treatment of vertebral compression fractures (VCFs). The transpedicular approach has been utilized for more than 3 decades for vertebral augmentation due to VCFs. Vertebroplasty has been expanded with the advent of balloon kyphoplasty (BKP) introduced in late 1990s (8). Kyphoplasty or mechanical vertebral augmentation now includes devices with larger instrumentation such as Kiva (Benvenue Medical, Santa Clara, CA) designed to improve upon vertebroplasty by incorporating a balloon tamp or other reduction device with or without an intrabody vertebral implant designed to facilitate height restoration of the VCF as well as safe and effective vertebral augmentation (9-15).

Additional access methods adding to the standard transpedicular approach to the vertebral bodies have been described. In 2007 the parapedicular approach to the thoracic and lumbar vertebral bodies was discussed and shown to be a safe and reliable alternative (16).

Potential problems arise with all of the above access techniques when the approach is obstructed by hardware from prior surgery, inhibiting safe access to the desired area of the vertebral body. The desires to use larger instruments, place an intrabody vertebral implant, or access the inferior portion of a vertebral body that is highly compressed are also specific scenarios that are often not adequately addressed by the transpedicular route to the vertebral body.

Potential complications to the modified inferior endplate approach are similar to that of other access techniques. They include injury to surrounding organs, hematoma formation, rib fractures, nerve root injury, and vascular injury $(17,18)$. In this article we will demonstrate the modified inferior endplate approach anatomy and show that it is a viable and reliable alternative for access to the vertebral bodies. We will present the results we have found from cadaver dissection and report complication rates associated with the modified inferior endplate approach.

\section{Methods}

\section{Patients}

A total of 96 vertebral compression fractures from T5 to L5 were treated by the modified extrapedicular endplate approach, performed by the senior author between July 2008 and August 2012. There were 72 patients between ages 27 and 98 (mean age 73.2 years) who underwent vertebral compression fracture treatment. Indications for this extrapedicular approach included patients with existing and obstructing spinal hardware at the vertebral level to be treated (11 patients), patients with severe vertebral compression of more than 70 percent loss of vertebral body height (17 patients), and fractures treated with access instruments larger than 11 gauge or $3.048 \mathrm{~mm}$ (68 patients). All patients underwent preoperative imaging assessment using a combination of conventional radiography, magnetic resonance imaging (MRI), computed tomography $(C T)$, and nuclear scintigraphy. The conventional workup for patients with VCFs consists of MRI for those patients who are able to undergo this examination and a combination of CT and nuclear scintigraphy for those patients who have contraindications to MRI. Conventional radiographs were also obtained on a minority of the patients. Informed consent and institutional review board approval were obtained.

All patients undergoing vertebral augmentation had a physical examination prior to their VCF treatment. Their strength, neurological status, and musculature were assessed and compared to the post-operative evaluation. All patients received a post-procedure CT and were either kept overnight and released the following day or discharged the same day provided they met the appropriate discharge criteria.

\section{Technical Description of Inferior Endplate Approach}

After the physical examination and obtaining informed consent, patients are placed prone on the operating room table where they are sterilely prepped and draped. Conscious sedation is then titrated with a combination of midazolam and fentanyl in $1 \mathrm{mg}$ and 50 mcg increments, respectively, and titrated to effect with ketamine added in 25 to 50 mg increments as needed. A sedation nurse monitors blood pressure and oxygen saturation and administers oxygen via a vented oxygen mask. A combination of antibiotics is given, including a 


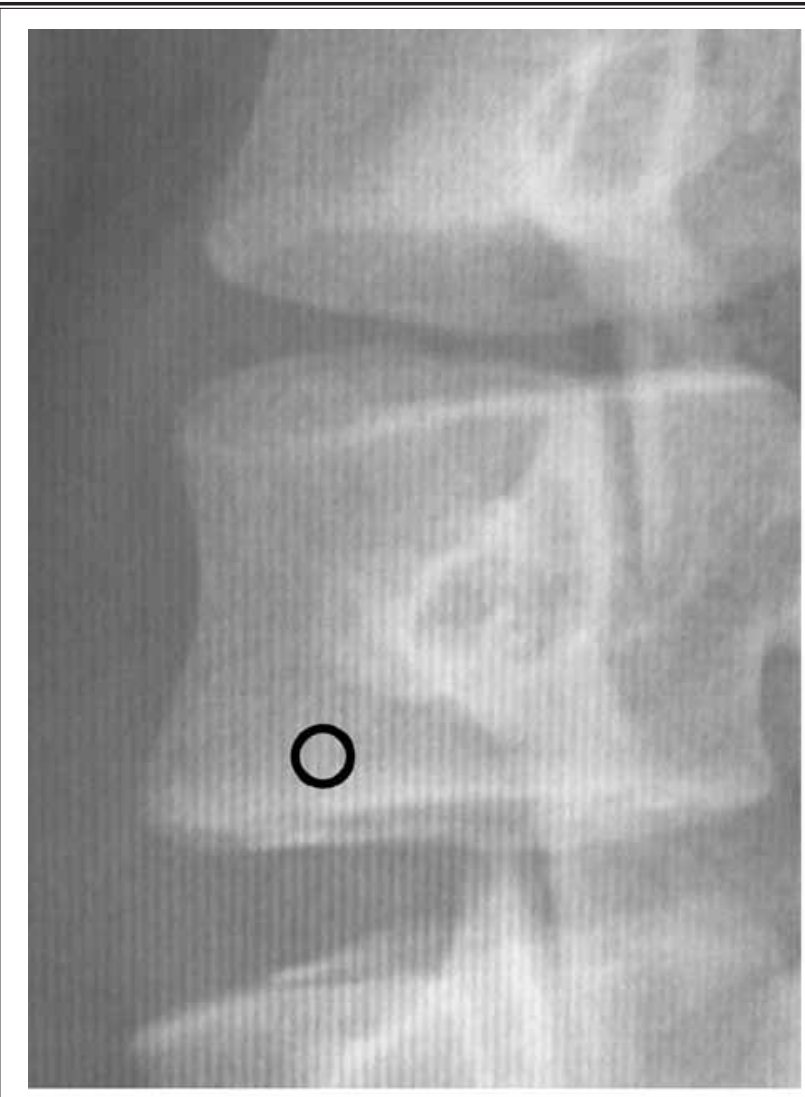

a.

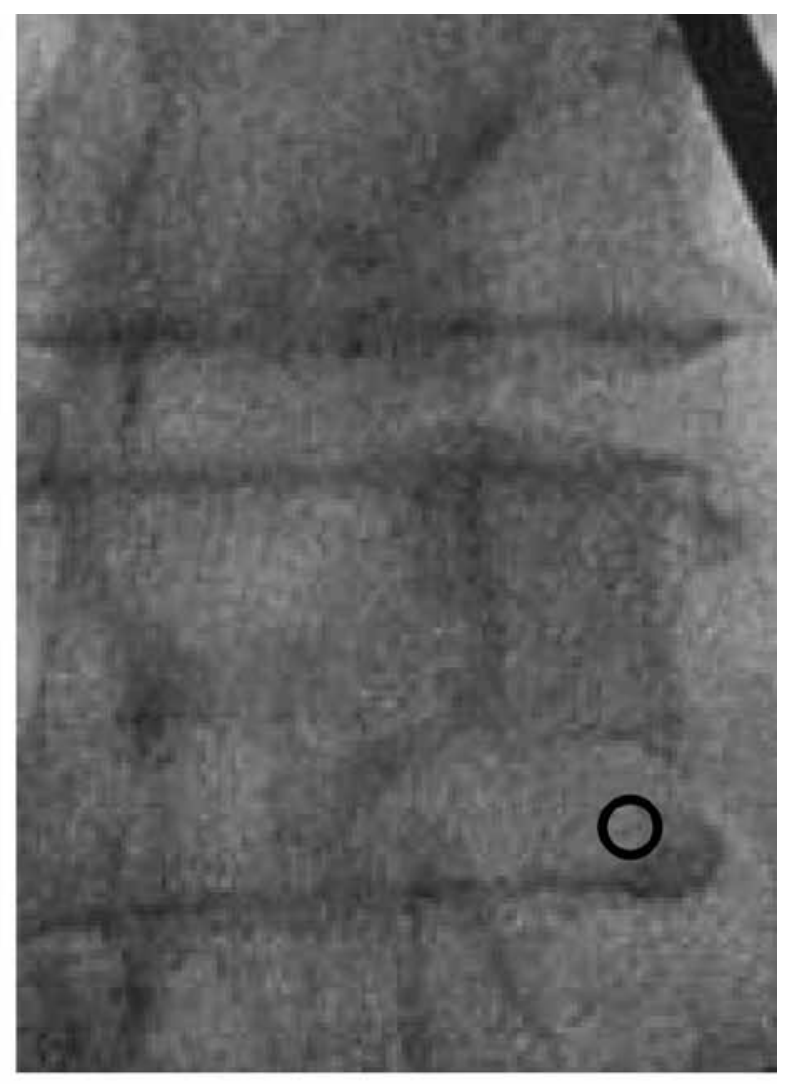

b.

Figs. 1a and 1b. Oblique fluoroscopic views of the lumbar spine (a) and the thoracic spine (b) obtained at 45 degrees and 30 degrees off midline respectively. The initial vertebral body entry point is identified just anterior to the pedicle and above the inferior endplate (black circles in both images).

single periprocedural dose of $1 \mathrm{gm}$ of cefazolin and 200 $\mathrm{mg}$ of gentamycin. A single $600 \mathrm{mg}$ dose of Clindamycin is given for patients that have a previous documented reaction to penicillin.

The affected vertebral level is then identified using fluoroscopy (Ziehm, Orlando, FL), and the image intensifier (II) is positioned to view the vertebral body in a direct posteroanterior (PA) orientation with the endplates parallel to the $\mathrm{x}$-ray beam and the spinous process centered between the pedicles. The side of entry is chosen by the physician performing the procedure based on the patient's anatomy and the type of fluoroscopy equipment utilized. The entry point should be marked on the skin in the area superior to the inferior endplate just anterior to the pedicle at an angle of 45 degrees on the ipsilateral side of placement in the lumbar spine and 30 degrees in the thoracic spine (Figs. 1a and 1b). At this point the skin and subcuta- neous tissues are anesthetized with 3 to $5 \mathrm{~mL}$ of $2 \%$ lidocaine. A small incision is made in this location and the needle is inserted into the target area while being stabilized by a Kocher clamp or other stabilizing device. Immediately after inserting the needle the proceduralist can rotate the II to the side of the needle to the $45 / 30$ degree angle to assess the trajectory of the needle. Anteroposterior (AP) and lateral views are also helpful for assessing the position of the needle tip (Figs. 2a and 2b). The entry point of the needle is $0.5-1.0 \mathrm{~cm}$ above the inferior endplate anterior to the ipsilateral pedicle, and the needle should be kept horizontal in an axial plane while being advanced. The needle is advanced into the outermost vertebral cortex of the vertebral body by tapping with a mallet. At this point the II is adjusted to the lateral position to ensure correct needle tip placement. Continue to advance needle with the mallet until the needle tip is placed in 

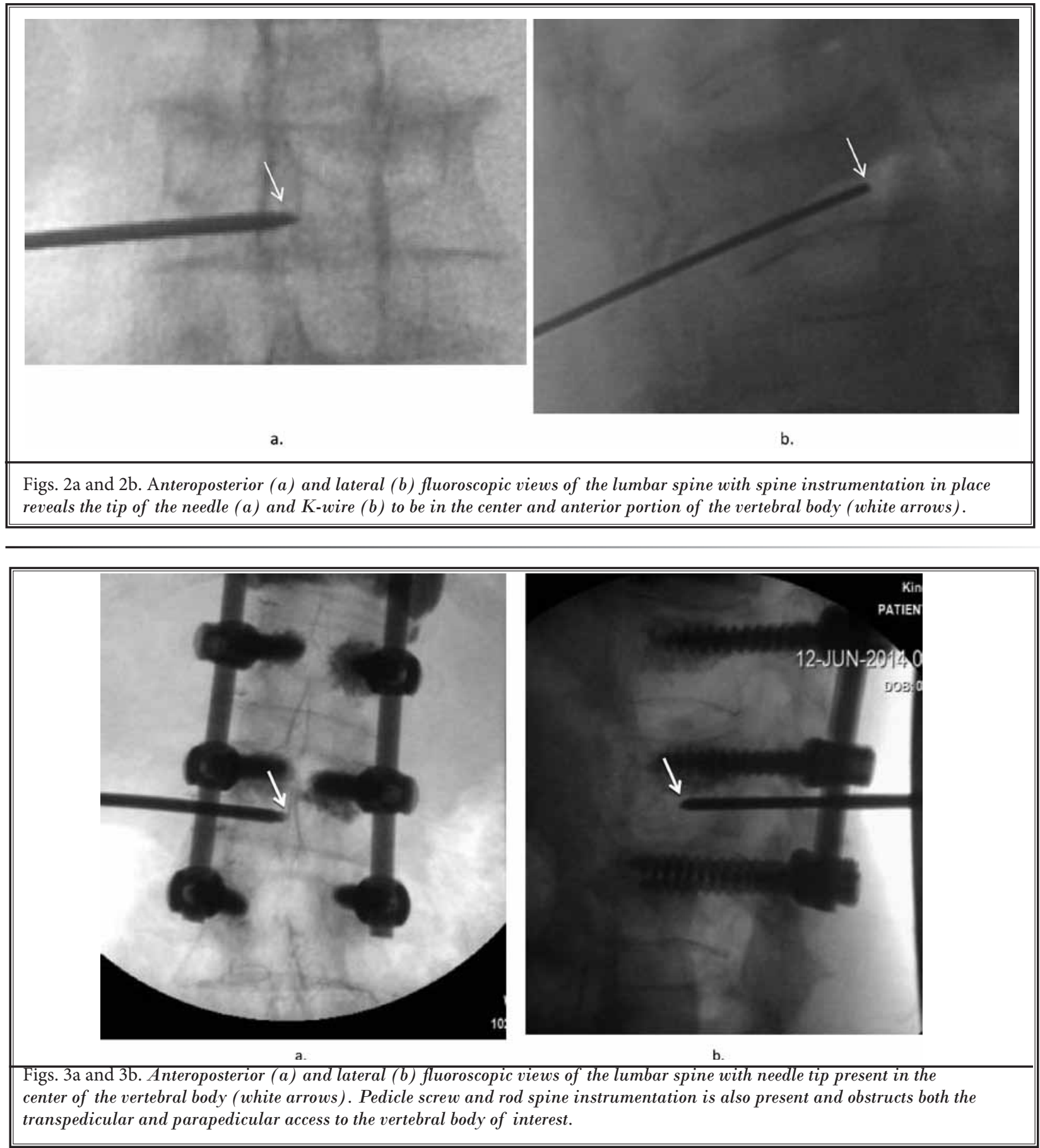

the middle to anterior third of the vertebral body (Figs. $2 \mathrm{a}$ and $2 \mathrm{~b}$ ). A combination of AP and lateral views are used to adjust the needle so that the needle tip is in the center position of the vertebral body (Figs. 3a and 3b).
In this patient cohort polymethylmethacrylate (PMMA) was injected in all vertebral augmentation cases with injected volumes that were similar to that used in transpedicular vertebral body stabilization procedures. 
Fig. 4. a. Lateral view of a cadaver lumbar spine shows the neurologic, soft tissue, and osseous structures (white arrows) labeled by name. The border of the safe vertebral body entry zone is present at the inferior and mid-posterior portion of the vertebral body (shown by the area within the trapezoid shaped black dotted line). b. Lateral x-ray view of the lumbar spine shows the radiographic imaging correlate to the cadaver representation of the safe vertebral body entry zone (shown by the area within the trapezoid shaped dotted line).

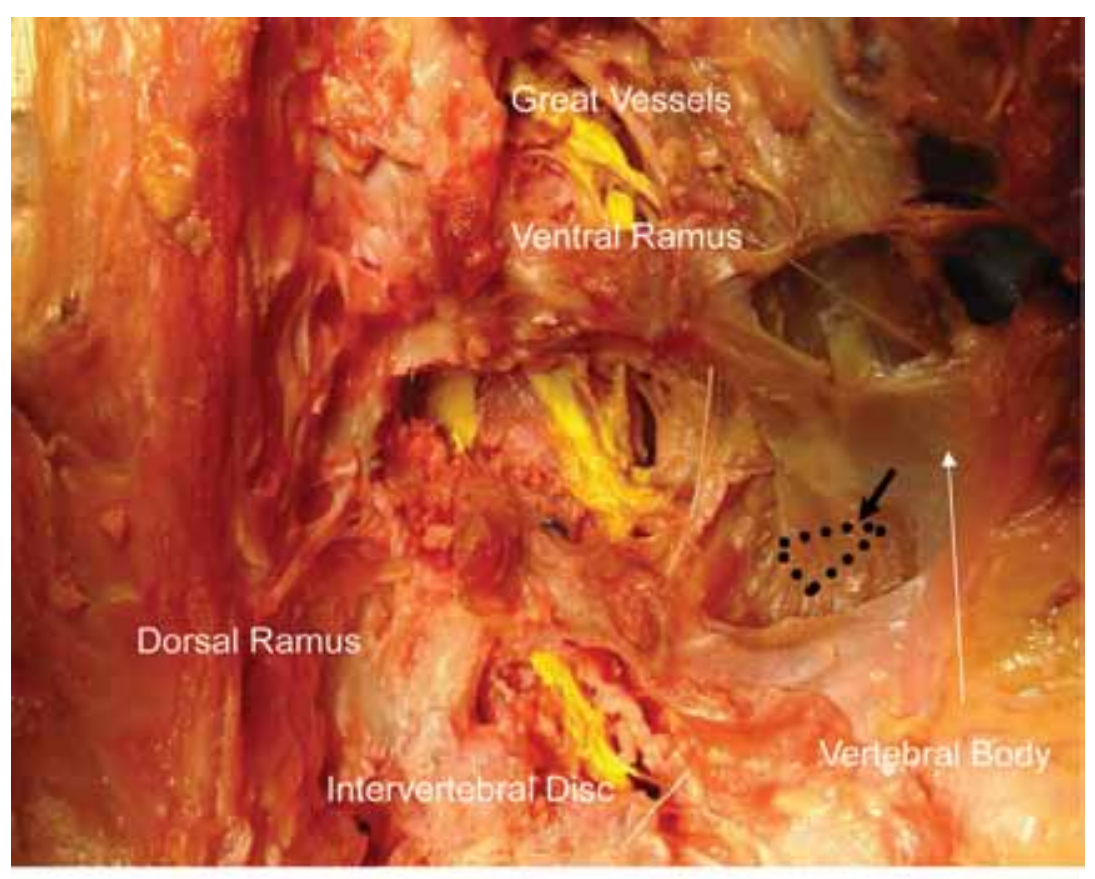

\section{Aatomic Correlation to Inferior Endplate Approach}

The exiting and traversing nerve roots and the exiting spinal nerve root quickly divide into the ventral and dorsal rami (Fig. 4a). The ventral ramus courses inferiorly and obliquely across the lateral and posterior margin of the vertebral body. The ventral ramus runs more horizontally in the thoracic spine than in the lumbar spine. There are neurologic connections between the ventral rami and the sympathetic chain which is present at T4-T12 on the left via the rami communicantes. They receive the postganglionic non-myelinated fibers from the sympathetic trunk. The fibers then join both the dorsal and ventral rami and supply the organs.

The entry target for the modified inferior endplate extrapedicular approach is just anterior to the pedicle and $3-5 \mathrm{~mm}$ above the inferior endplate when the vertebral body is viewed ipsilaterally 30 degrees off midline. In the thoracic spine, the needle entry point is just anterior to the exiting ventral rami and superior to the ventral ramus of the vertebral body (i.e., at T8, the entry point will be superior to the ventral ramus of T8). The needle penetrates the inferolateral portion of the vertebrae just above the inferior endplate where the cortex is typically not very thick. In the thoracic spine care must be taken to avoid contact with the pleura which, in cadaveric studies, has been found to be immediately adjacent to the inferolateral pedicle (19-20).

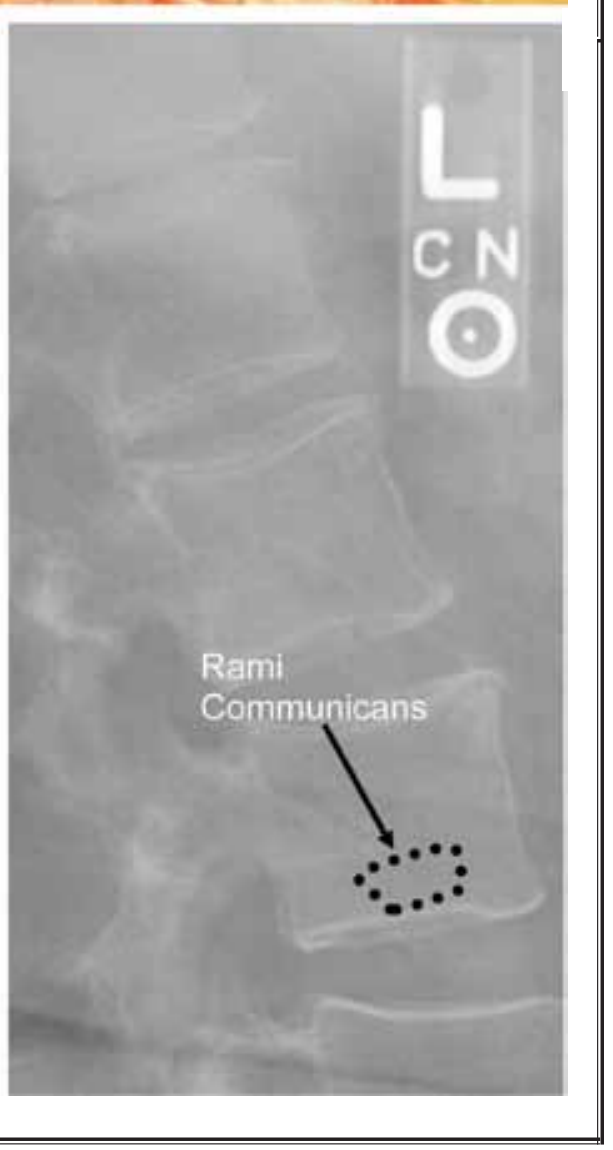




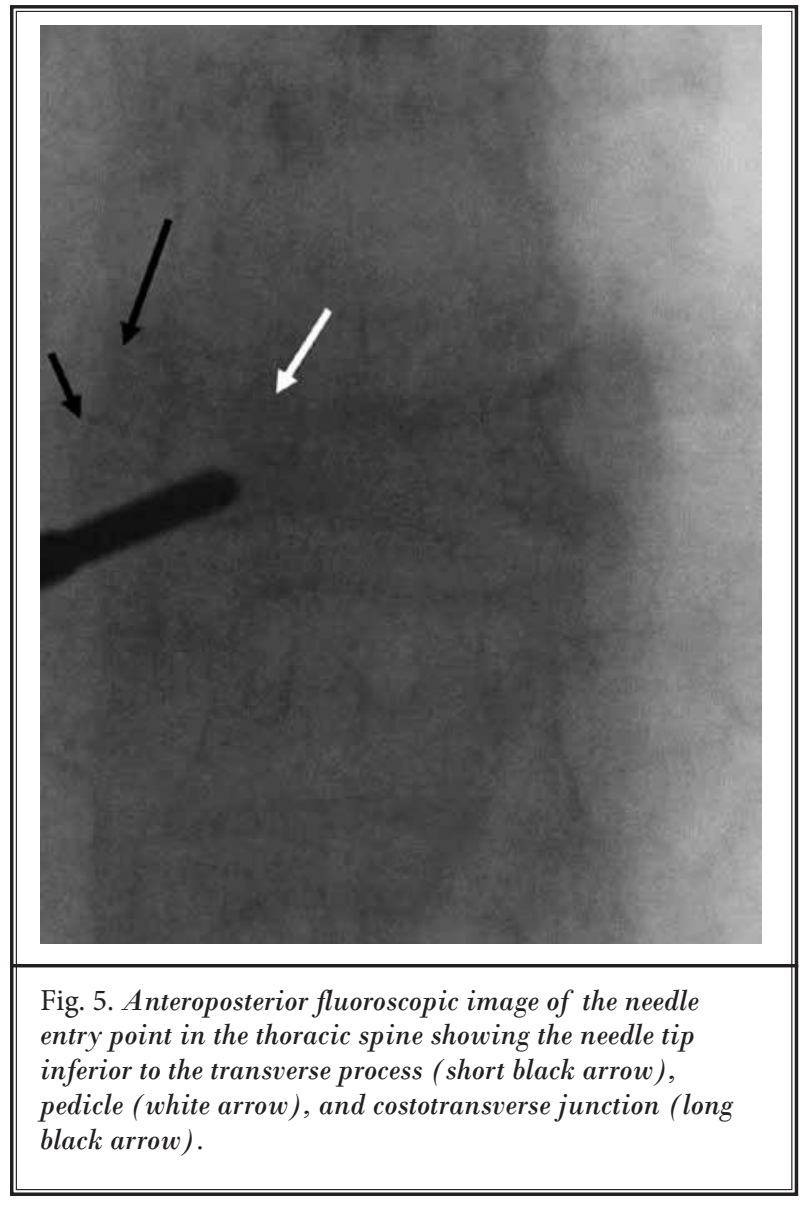

This can be done by keeping the needle trajectory flat (much greater than 30 degrees off midline) just after it is inserted into the soft tissue until the needle is near the spine and away from the pleura. This angle of entering the vertebral body is effective at avoiding the dorsal nerve root ganglion that is located posterosuperior to the entry point, the aorta (T5-T10 on the left), and the azygos vein (T5-T11 on the right). The trajectory allows the needle to pass inferior to the transverse process, costotransverse junction, and pedicle (Fig. 5). The lack of obstructing osseous structures provides unfettered access to the vertebral body.

In the lumbar spine, the ventral rami course more vertically and the needle entry point is anterior to the exiting ventral rami. The optimal entry point in the lumbar spine, as in the thoracic spine, is just above the inferior endplate just anterior to the pedicle when the vertebral body is viewed 45 degrees ipsilateral off midline. The needle entry point is in the inferior and lateral portion of the vertebral body. The needles trajectory is horizontal and well anterior of the descending ventral rami.

\section{Results}

After cadaver dissection demonstrating that the inferior portion of the vertebral body anterior to the ipsilateral pedicle and superior to the inferior endplate was relatively avascular and aneural, a total of 96 vertebral fractures were treated using the extrapedicular technique described above. This represented a total of $7.4 \%$ of the total number of patients treated during this time period. There were no known clinical complications from either the needle access or the instrumentation used to perform the vertebral augmentation with this technique.

\section{Discussion}

Limitations of typical transpedicular access to the vertebral body include spinal hardware, indistinct visualization of the pedicle, severely compressed vertebral bodies, and instrumentation that is too large to perform a transpedicular approach. The parapedicular route may also have limitations based on these factors. The inferior portion of the vertebral body is typically not occupied by spine hardware as the conventional placement of pedicle screws is through the longitudinal access of the pedicle with a slightly lateral to medial and axial orientation. The position of the screws is normally in the superior third to half of the vertebral body leaving the inferior half easily accessible via the modified inferior endplate approach (Fig. 6). Highly compressed anterior vertebral body wedge fractures can also have limitations with traditional access. The inferior endplate approach allows for penetration to the remaining intact vertebral body (Fig. 7).

The extrapedicular approach is not as commonly used to access the vertebral body due to a perceived higher risk of complications and from clinicians' lack of familiarity of the approach technique. Despite this higher risk perception, the rate of clinically significant complications is low and was nonexistent in our patient group. Injury to the segmental vertebral body artery has been reported with an extrapedicular approach but the entry point of this approach was more superior and lateral to the pedicle than inferior to the pedicle and above the inferior endplate (21). Many techniques described as extrapedicular are around the pedicle, often enter at the vertebral body-pedicle junction, and are probably better described as parapedicular 


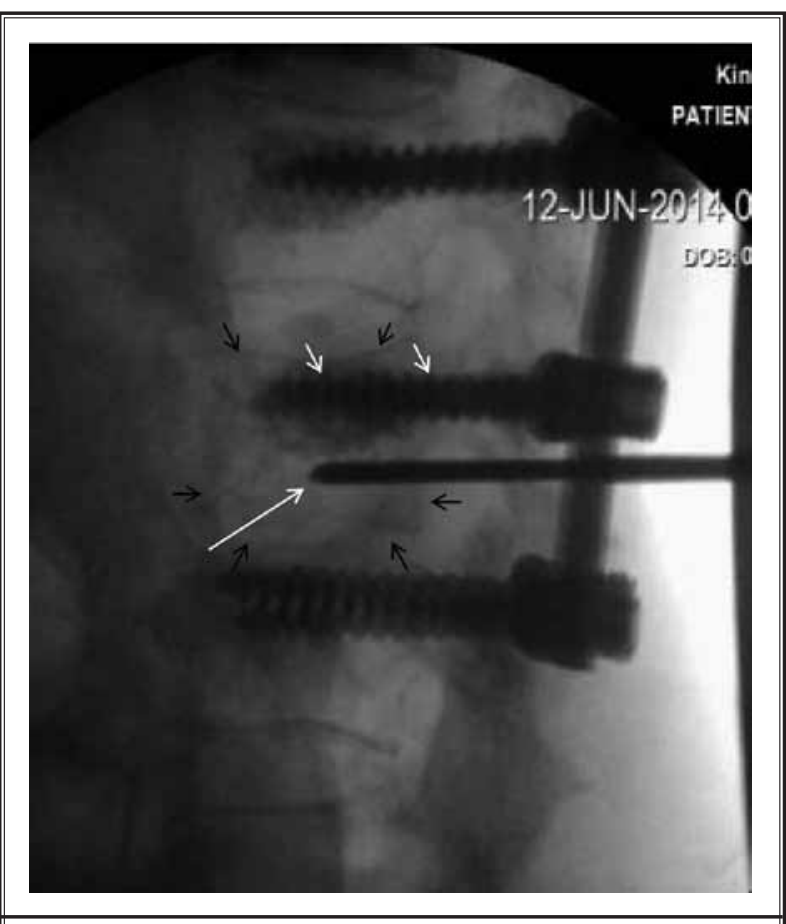

Fig. 6. Lateral fluoroscopic image of the lumbar spine demonstrating the placement of the needle (long white arrow) inferior to the pedicle screws into vertebral body (short white arrows). This image demonstrates the use of the inferior endplate approach to penetrate the vertebral body (short black arrows) without interference of the pedicle screws.

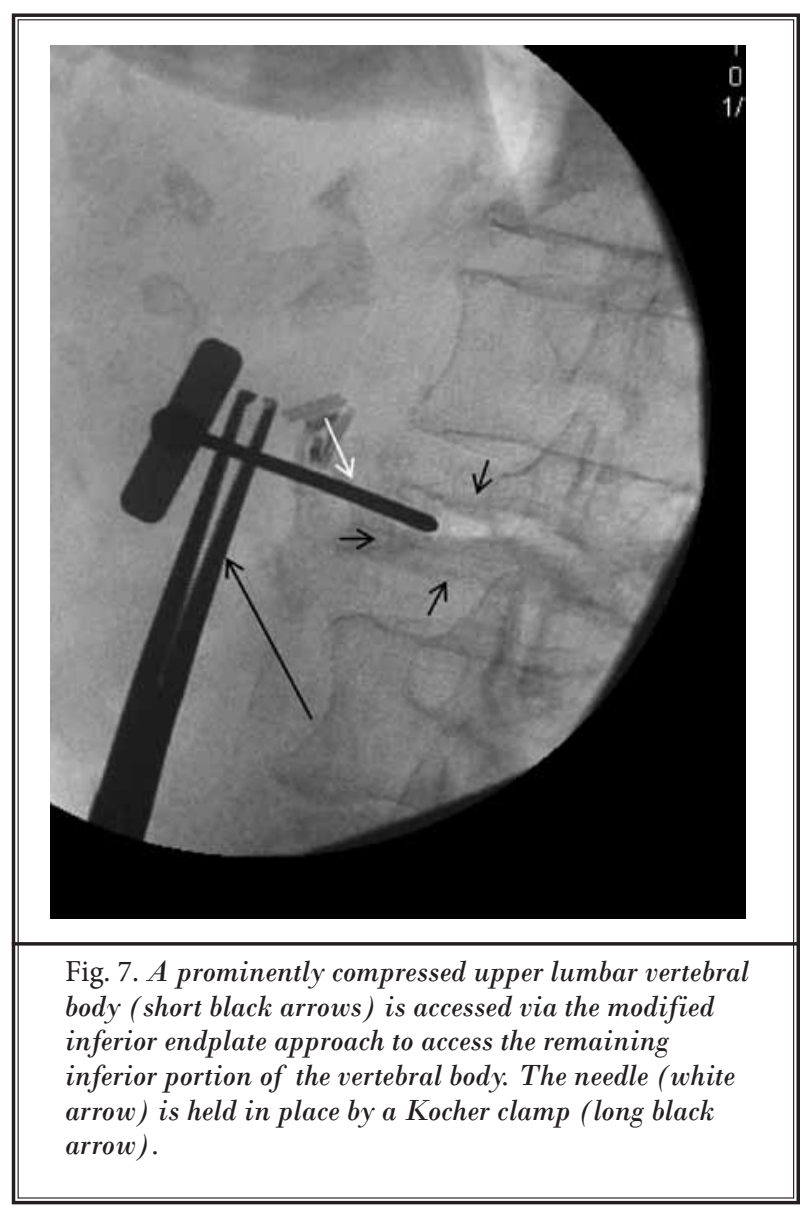

performing this access to the vertebral body. In the thoracic spine, starting with the needle more shallow or horizontally oriented until it touches the inferior portion of the vertebral body avoids contact with the pleura which can be immediately adjacent to the inferolateral pedicle based on cadaveric studies (20). Also in the thoracic spine, entering the vertebral body just anterior to the pedicle avoids the aorta at T5-T10 on the left side and the azygous vein at T5-T11 on the right side. In both the thoracic and lumbar spine, entering the inferior portion of the vertebral body just anterior to the pedicle as seen on the oblique view avoids the dorsal and ventral rami and dorsal nerve root ganglion.

The paravertebral arteries and veins in the lumbar spine are naturally larger than those found in the thoracic spine. These vessels are located near the mid portion of the vertebral body, and the arterial location and vessel size are more predictable than the corresponding venous structures. If the appropriate inferior approach is maintained, the posterior entry point will 
be inferior to the majority of the paravertebral vessels in a relatively avascular region. The psoas musculature lies immediately adjacent to the vertebral bodies as was seen during the manual cadaver dissection of these muscles. This close muscular attachment likely limits hemorrhage and subsequent hematoma formation from the paravertebral access point by providing anatomic tamponade.

In addition to the points discussed above, there are other key points to a successful approach to the thoracic and lumbar vertebral bodies that should be considered when this approach is applied. Prior to obtaining the oblique view, the vertebral body should always be lined up in a direct AP view before arcing 45 degrees off midline in the lumbar spine and 30 degrees in the thoracic spine to ensure the entry is at the appropriate angle. After the appropriate obliquity is obtained, the skin entry point is marked, local anesthesia applied, and the needle is inserted. When initially positioning needle for the approach into the vertebral body, use a Kocher clamp or other instrument to hold the needle as it will not be particularly stable within the surrounding soft tissue (Fig. 7).

The limitations of the modified inferior endplate extrapedicular access include the ability to access the upper thoracic spine and the lower lumbar spine, specifically the L5 vertebral body. The superoinferior angulation of the ribs occasionally limits the access to the thoracic spine entry point by overlying the appropriate pathway when the vertebral body is viewed at 30 degrees from midline. The rib angulation will also occasionally require the needle to pass under the rib to reach the target point. The inferior margin of the rib is to be avoided as that is the location of its neurovascular bundle. The wide pedicles at the L5 level make the transpedicular approach the naturally favored method for accessing the L5 vertebral body. The modified inferior endplate extrapedicular access at this level is mostly difficult or impossible due to the challenge of navigating around the large pedicles and over the superior margin of the iliac wing.

We have performed the modified inferior endplate extrapedicular approach in 96 patients with vertebral compression fractures from July 2008 to August 2012 including 5 Spinewave Staxx procedures where a $4 \mathrm{~mm}$ $x 11 \mathrm{~mm}$ cannula was placed into the vertebral body with this approach and 32 cases of vertebral augmentation with allograft bone using a $10 \mathrm{~mm}$ cannula. No complications (nerve root injuries, hematomas, or injuries to spinal canal contents) were identified in any of these patients undergoing this procedure. No cases were identified where the PMMA extruded proximally around the needle or working channel at its entry point.

\section{Conclusions}

The modified inferior endplate approach may be used as an alternative access technique when the proceduralist is faced with obstructions in the typical access routes, when the vertebral body is very compressed superiorly or when a device larger than the size of the pedicle is inserted. The needle trajectory can be easily planned using anatomical landmarks under fluoroscopic guidance. The modified inferior endplate approach, like the parapedicular and transpedicular approaches, offers a favorable route to the vertebral body that is relatively avascular and aneural. When the vertebral level is limited by hardware, the vertebral body is severely compressed, or there is a need for use of large devices, our experience supports the use of the inferior endplate approach as one that is reproducible, reliable, and associated with a negligible complication rate.

\section{References}

1. Peh W, Munk P, Gilula L. Percutaneous vertebral augmentation: Vertebroplasty, kyphoplasty and skyphoplasty. Radiologic Clinics of North America 2008; 46:611-635.

2. Denaro L, Longo UG, Denaro V. Vertebroplasty and kyphoplasty: Reasons for concern? Orthopedic Clinics of North America. 2009; 40:465-471.

3. Levy H, Seydafkan S, Rice JD, Easley KA, Tangpricha V. Comparative efficacy of vertebroplasty, kyphoplasty and medical therapy for vertebral fractures on surviv- al and prevention of recurrent fractures. Endocrine Practice 2012; 18:499-507.

4. Hao J, Hu Z. Percutaneous cement vertebroplasty in the treatment of symptomatic vertebral hemangiomas. Pain Physician 2012; 15:43-49.

5. Roy-Camille R, Saillant G, Mamoudy P, Leonard P. [Biopsy of the vertebral body using a posterior transpedicular approach]. Rev Chir Orthop Reparatrice Appar Mot 1983; 69:147-149.

6. Ringer AJ, Bhamidipaty SV. Percutaneous access to the vertebral bodies: A video and fluoroscopic overview of access techniques for trans-, extra-, and infrapedicular approaches. World Neurosurg 2013; 80:428-435. doi: 10.1016/j. wneu.2012.09.005. Epub 2012 Sep 23.

7. Marlin E, Nathoo N, Mendel E. Use of percutaneous kyphoplasty and vertebroplasty in spinal surgery. J Neurosurg Sci 2012; 56:105-112.

8. Lane JM, Girardi F, Parvaianen H. Preliminary outcomes of the first 226 consecutive kyphoplasties for the fixation of painful osteoporotic vertebral compres- 
sion fractures [abstract]. Osteoporosis Int 2000; 11:S206.

9. Anselmetti GC, Tutton SM, Facchini FR, Miller LE, Block JE. Percutaneous vertebral augmentation for painful osteolytic vertebral metastasis: A case report. Int Med Case Rep J 2012; 20:13-17.

10. Anselmetti GC, Manca A, Tutton S, Chiara G, Kelekis A, Facchini FR, Russo F, Regge D, Montemurro F. Percutaneous vertebral augmentation assisted by PEEK implant in painful osteolytic vertebral metastasis involving the vertebral wall: Experience on 40 patients. Pain Physician 2013; 16:E397-E404.

11. Korovesis P, Vardakastanis K, Repantis T, Vitsas V. Transpedicular vertebral body augmentation reinforced with pedicle screw fixation in fresh traumatic $A_{2}$ and $A_{3}$ lumbar fractures: Comparison between two devices and two bone cements. Eur] Orthop Surg Traumatol 2013; Aug 28 [Epub ahead of print].

12. Korovesis P, Vardakastanis K, Repantis $\mathrm{T}$, Vitsas V. Balloon kyphoplasty versus KIVA vertebral augmentation - comparison of 2 techniques for osteoporotic vertebral body fractures. Spine 2013; 38:292-299.

13. Korovesis P, Repantis T, Miller LE, Block JE. Initial clinical experience with a novel vertebral augmentation system for treatment of symptomatic vertebral compression fractures: A case series of 26 consecutive patients. BMC Musculoskelet Disord 2011; 12:206.

14. Otten LA, Bornemnn R, Jansen TR, Kabir K, Pennekamp PH, Wirtz DC, Stu- we B, Pflugmacher R. Comparison of balloon kyphoplasty with the new Kiva ${ }^{\circledR}$ VCF System for the treatment of vertebral compression fractures. Pain Physician 2013; 16: $\mathrm{E}_{505}-\mathrm{E}_{512}$.

15. Panagiotis $\mathrm{K}$, Konstantinos $\mathrm{V}$, Vasilios $\mathrm{V}$, Vasilios S. Is Kiva implant advantageous to balloon kyphoplasty in treating osteolytic metastasis to the spine? Comparison of 2 percutaneous minimal invasive spine techniques: A prospective randomized controlled short-term study. Spine. 2014; 39:E231-E239.

16. Beall D, Braswell J, Martin H, Stapp A, Puckett T, Stechison M.T. Technical strategies and anatomic considerations for parapedicular access to thoracic and lumbar vertebral bodies. Skeletal Radiology 2007; 36:47-52.

17. Lonstein J, Denis F, Perra J, Pinto M, Smith M, Winter R. Complications associated with pedicle screws. The Journal of Bone and Joint Surgery 1999; 81:1519-1528.

18. Al-Nakshabandi NA. Percutaneous vertebroplasty complications. Ann Saudi Med 2011; 31:294-297.

19. Vaccaro AR, Rizzolo SJ, Allardyce TJ, Ramsey M, Salvo J, Balderston RA, Cotler JM. Placement of pedicle screws in the thoracic spine. Part I: Morphometric analysis of the thoracic vertebrae.J Bone Joint Surg Am 1995; 77:1193-1199.

20. Vaccaro AR, Rizzolo SJ, Balderston RA, Allardyce TJ, Garfin SR, Dolinskas C, An HS. Placement of pedicle screws in the thoracic spine. Part II: An anatomical and radiographic assessment. J Bone Joint Surg Am 1995; 77:1200-1206.
21. Heo DH, Cho YJ. Segmental artery injury following percutaneous vertebroplasty using extrapedicular approach. J Korean Neurosurg Soc 2011; 49:131-133. doi: 10.3340/jkns.2011.49.2.131.

22. Ryu KS, Huh HY, Jun SC, Park CK. Single-balloon kyphoplasty in osteoporotic vertebral compression fractures: Far-lateral extrapedicular approach. J Korean Neurosurg Soc 2009; 45:122-126. doi: 10.3340/jkns.2009.45.2.122.

23. Kim HY, Kim SW, Ju Cl. Balloon kyphoplasty through extrapedicular approach in the treatment of middle thoracic osteoporotic compression fracture: T5-T8 level. J Korean Neurosurg Soc 2007; 42:363-366. doi: 10.3340/ jkns.2007.42.5.363.

24. Kang JH, Kuh SU, Shin ZZ, Cho YE, Yoon YS, Chin DK. The effectiveness of osteoplasty system (vertebroplasty using large cannula-low pressure delivery system) in compression fracture and other spinal pathology.] Korean Neurosurg Soc 2005; 38:259-264.

25. Lee KS, Cho YJ, Choi JH, Cho SM, Ryu KS, Ahn MS. A small amount of bone cement injected vertebroplasty utilizing percutaneous vertebral body access technique for osteoporotic vertebral fractures. Korean J Spine 2005; 2:333-341.

26. Cho YJ, Choi JH, Cho SM. Vertebroplasty utilizing percutaneous vertebral body access (PVBA) technique for osteoporotic vertebral compression fractures in the middle thoracic vertebrae. J Korean Neurosurg Soc 2007; 41:161-165. 
